\title{
HIPERTENSÃO OCULAR SECUNDÁRIA À LUXAÇÃO DE CRISTALINO PARA CÂMARA ANTERIOR NA SÍNDROME DE MARFAN
}

\author{
Daniel Prado Beraldo ${ }^{1}$, Fernando Buzatto Mantovan ${ }^{2}$, Emanuele Moraes Mello ${ }^{2}$ \\ ${ }^{1}$ Hospital Regional de Presidente Prudente - HRPP, Presidente Prudente, SP, ${ }^{2}$ Universidade do Oeste Paulista - \\ UNOESTE, Presidente Prudente, SP. E-mail: danielpberaldo@hotmail.com
}

\begin{abstract}
RESUMO
A síndrome de Marfan (SMF) é uma rara desordem metabólica autossômica dominante, causada por mutações no gene responsável pela codificação da proteína fibrilina-1. Caracteriza-se por manifestações cardiovasculares, musculoesqueléticas e oculares. O objetivo desse relato foi apresentar uma complicação oftalmológica rara em paciente portadora da SMF. Feminino, 51 anos, compareceu ao pronto socorro com queixa de dor intensa, baixa acuidade visual (AV) e hiperemia em olho direito (OD), há dois dias. Biomicroscopia: ectopia do cristalino (luxação) para câmara anterior; Tonometria: $56 \mathrm{mmHg}$. Foi prescrito medicamentos hipotensores, que reduziram a pressão intraocular (PIO) para $8 \mathrm{mmHg}$. Encaminhada para cirurgia de urgência (facectomia sem implante de LIO) a fim de evitar maiores danos à córnea e ao nervo óptico. No pós operatório, apresentou controle da PIO e melhora da AV para 20/50. Na SMF se faz necessário o acompanhamento multidisciplinar, possibilitando diagnósticos precoces, o que pode prevenir e minimizar eventuais complicações.
\end{abstract}

Palavras chave: síndrome de Marfan, ectopia do cristalino, hipertensão ocular, câmara anterior.

\section{OCULAR HYPERTENSION SECONDARY TO LENS DISLOCATION FOR ANTERIOR CHAMBER IN MARFAN SYNDROME}

\begin{abstract}
Marfan syndrome (MFS) is a rare autosomal dominant metabolic disorder, caused by mutations in the gene responsible for coding the fibrillin-1 protein. It is characterized by cardiovascular, musculoskeletal and ocular manifestations. The purpose of this report was to present a rare ophthalmologic complication in a patient with MFS. Female, 51 years old, attended the emergency room complaining of severe pain, low visual acuity and hyperemia in the right eye, started two days before. Biomicroscopy: ectopia lentis (luxated) for anterior chamber; Tonometry: $56 \mathrm{mmHg}$. Hypotensive drugs were prescribed which reduced intraocular pressure (IOP) to $8 \mathrm{mmHg}$. Referred for emergency surgery (facectomy without IOL implantation) in order to avoid further damage to the cornea and optic nerve. In the post operative period, presented IOP control and improved VA to 20/50. Multidisciplinary follow-up is necessary in patients with MFS, enabling early diagnosis, which can prevent and minimize any complications.

Keywords: Marfan syndrome, ectopia lentis, ocular hypertension, anterior chamber.
\end{abstract}

\section{INTRODUÇÃO}

A síndrome de Marfan (SMF) é uma doença genética do tecido conjuntivo descrita pelo pediatra francês, Antoine Bernard-Jean Marfan, em 1896. Em 1955, o quadro foi incluído por McKusick no grupo das doenças hereditárias do tecido conjuntivo. ${ }^{1}$ Estimativas históricas relatam prevalência de 1/5.000 indivíduos. ${ }^{2}$ É uma doença de herança autossômica dominante e que afeta o tecido conjuntivo com manifestações fenotípicas, atingindo tipicamente os sistemas cardiovascular, esquelético e ocular, 
podendo também envolver o sistema nervoso central, o aparelho respiratório e a pele. ${ }^{1,3}$ Essa mutação pode ter sido herdada de um dos genitores, que pode ou não apresentar manifestações clínicas, ou ter acontecido, pela primeira vez, mutação nova. Uma vez que a mutação está presente, a chance de transmissão para os filhos é de $50 \%{ }^{1}$

O diagnóstico clínico foi estabelecido em 1986 de acordo com os critérios de Berlim, substituídos em 1996 pelos critérios Ghent, que em 2010 foram atualizados para a mais recente versão. $^{4}$

A SMF nem sempre manifesta os sinais e sintomas desde o nascimento, apesar de ser congênita. Enquanto algumas pessoas apresentam características de Marfan ao nascimento ou quando são crianças pequenas (incluindo condições graves, como o aumento da aorta), em outras, essas características apenas aparecem na fase adulta. Isso torna muito importante que as pessoas com SMF e condições relacionadas recebam diagnóstico e tratamento precisos e precoces, sem os quais se aumenta o risco de complicações potencialmente fatais.

A SMF é causada por mutações no gene responsável pela codificação de proteínas responsáveis pela formação de fibras elásticas e com função de suporte em alguns tecidos. Aquelas foram nomeadas fibrilina-1, gene localizado no cromossomo $15 .^{2}$ Expressam-se em grande parte do tecido conjuntivo ocular e foi investigada por técnicas de imunohistoquímica sendo encontrado uma ampla distribuição nesse tecido. ${ }^{5}$ A fibrilina é fisiologicamente ausente no vítreo e na retina. ${ }^{5}$

As moléculas de fibrilina ficam dispostas como contas de colar sobre o colágeno em um olho saudável. ${ }^{6} \mathrm{Na}$ SMF a fibrilina anormal acarreta deslizamento das bandas de colágeno, provocando alongamento da zônula e consequente retração da cápsula cristaliniana com alteração secundária da forma do cristalino, tornando-se mais globoso pela falta de tensão zonular sobre a cápsula. Neste caso, há menos processos ciliares, que apresentam orientação irregular e sinais de degeneração. Além do mais, a fibrilina anormal, localizada na região equatorial da cápsula do cristalino, leva a alterações no seu desenvolvimento e funcionamento. ${ }^{5}$

Dentre as manifestações oculares mais comuns, podem-se citar: a subluxação do cristalino, alta miopia e astigmatismo. Além disso, hipoplasia de íris, estrabismo, megalocórnea, córnea plana, catarata, glaucoma e descolamento de retina também podem ocorrer. ${ }^{3,7,8}$ Todos esses achados são importantes para auxiliar no diagnóstico precoce dos portadores da SMF.

A fibrilina é um componente de várias estruturas dos olhos, córnea, esclera, câmara anterior, úvea, fibras da zônula, cápsula cristaliniana e septo do nervo óptico. ${ }^{4} \mathrm{~A}$ alteração mais característica e mais frequente é a subluxação do cristalino (ectopia lentis), que varia de leve deslocamento superior e posterior até um grande deslocamento, onde a margem equatorial do cristalino pode ser vista na pupila cortando o eixo visual. Ectopia lentis (EL) foi relatada para fazer parte da SMF por Borger em $1914,{ }^{9}$ e foi relatado como a causa mais prevalente de EL por Fuchs \& Rosenberg em 1998. ${ }^{10}$ A subluxação é lentamente progressiva e torna-se clinicamente detectável na infância ou adolescência. Cerca de 60 a $80 \%$ dos pacientes desenvolvem subluxação do cristalino. $^{11}$ As zônulas são frequentemente alongadas, mas intactas, e a iridodonese e a facodonese são aparentes durante o exame com a lâmpada de fenda. ${ }^{12}$ Pode variar de um leve deslocamento assintomático visto apenas na dilatação póspupilar a uma subluxação significativa que coloca o equador do cristalino no eixo pupilar e causa diplopia monocular. O deslocamento é geralmente superotemporal, no entanto, um deslocamento anterior do cristalino para dentro da pupila ou da câmara anterior pode causar bloqueio pupilar com o desenvolvimento subsequente de glaucoma agudo ou glaucoma crônico de ângulo fechado, enquanto luxações posteriores podem causar tração vítrea na retina, levando a vitreíte crônica e inflamação coriorretiniana. ${ }^{13,14}$ Quando o diagnóstico de subluxação do cristalino é feito antes dos outros comprometimentos sistêmicos, deve-se pensar na hipótese de SMF. É necessário o acompanhamento multidisciplinar, pois outros comprometimentos clínicos da síndrome podem estar presentes. $^{2}$

Os principais diagnósticos diferenciais da SMF em relação a ectopia lentis hereditária são a síndrome de Weill-Marchesani e homocisteinúria. ${ }^{1}$

Por conseguinte, o objetivo desse relato é apresentar um caso peculiar, em que após ocorrido luxação de cristalino para a cavidade vítrea, fato relativamente trivial na SMF, 
apresentou, de forma espontânea e inesperada, o cristalino luxado para a câmara anterior, que resultou em hipertensão intraocular grave. Apesar das condições, a conduta do caso ocorreu sem intercorrências.

\section{RELATO DE CASO}

Relata-se um caso de uma paciente, do sexo feminino, 51 anos, branca, encaminhado ao Pronto Socorro Oftalmológico do Hospital Regional de Presidente Prudente devido ao quadro de dor intensa e baixa acuidade visual (BAV) progressiva e hiperemia em olho direito (OD), há dois dias. Negava qualquer tipo de trauma ou intercorrência. Apresentava diagnóstico de luxação completa do cristalino em OD para a cavidade vítrea há 2 meses, sendo acompanhada no ambulatório de retina (aguardava cirurgia de vitrectomia posterior) com acuidade visual (AV) de 20/40 em ambos olhos (AO), prévia ao quadro atual. O presente caso foi avaliado e aprovado pelo Comitê de Ética em Pesquisa (CEP) da UNOESTE, protocolo número 6621, e pela Plataforma Brasil, número CAAE. 40710820.0.0000.5515.
Antecedentes oftalmológico: OD: aguardava cirurgia eletiva de vitrectomia posterior (VVPP); OE: VVPP associado a facoemulsifação e implante de lente intraocular (LIO), iridotomia a laser e dois reposicionamentos de LIO.

Ao exame oftalmológico, a paciente apresentou AV corrigida em OD de movimento de mãos e em OE de 20/40. Na biomicroscopia, OD encontrava-se com conjuntiva hiperemiada $2+$, córnea íntegra com opacidade moderada difusa, edema corneano $3+$, câmara anterior formada, rasa e sem reação de câmara anterior, cristalino luxado para câmara anterior (Figura 1). Impossível avaliar a fundoscopia por opacidade de cristalino em olho direito. O OE estava calmo e com a LIO tópica. Fundoscopia OE: sem alterações. Pressão intraocular (PIO) Tonometria (Goldmann): OD: $56 \mathrm{mmHg}$; OE: 16 $\mathrm{mmHg}$. A medida do comprimento axial (AL), 2 meses prévia ao quadro atual, era de $26,39 \mathrm{~mm}$ (realizada usando ultrassom B-18, Apramed, Brasil). Não havia medida da câmara anterior naquela ocasião, pois a paciente encontrava-se afácica.

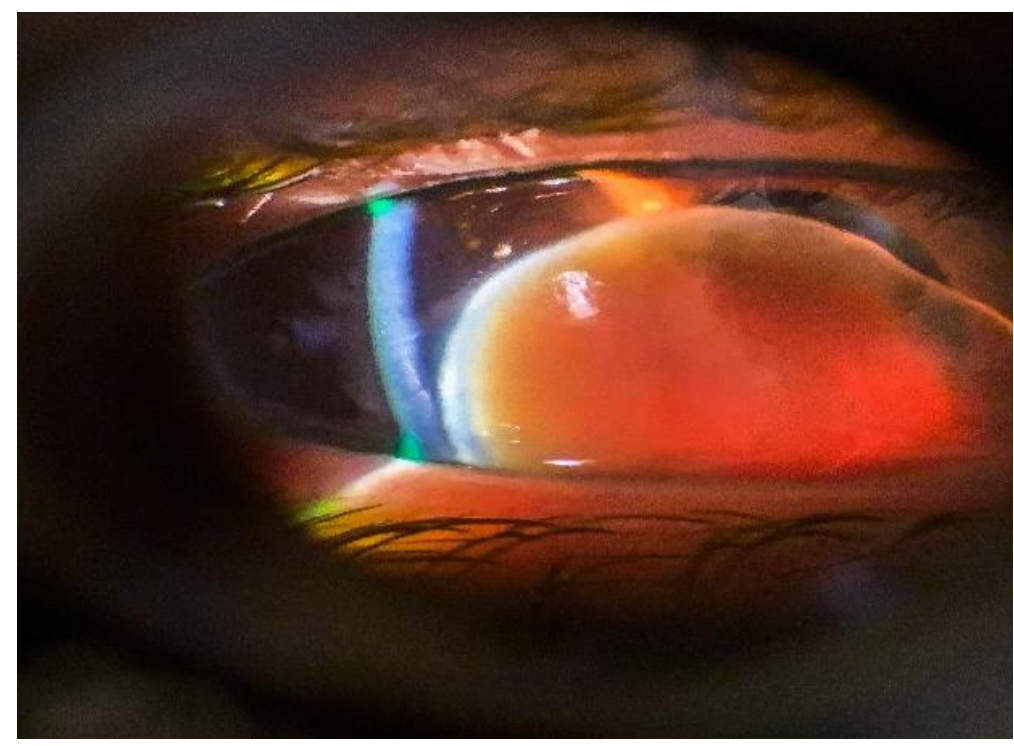

Figura 1. Luxação de cristalino para a câmara anterior. Fonte: $\mathrm{O}$ autor.

Foi instituído, imediatamente, o tratamento clínico com três classes de medicamentos hipotensores, corticóide tópico e acetazolamida via oral, que reduziu a PIO para 8 $\mathrm{mmHg}$. Tentativa infrutífera de decúbito dorsal para retorno do cristalino ao vítreo, com dilatação de pupila.
Foi encaminhada para cirurgia de urgência (facectomia sem implante de LIO) a fim de evitar maiores danos à córnea e ao nervo óptico. Procedimento realizado sem intercorrências. No primeiro dia pós operatório, apresentou controle da PIO e melhora da AV (conta dedos dois metros). A biomicroscopia 
mostrava conjuntiva hiperemiada, hemorragia subconjuntival, córnea com opacidade leve difusa, dobras de Descemet 2+, câmara anterior formada e profunda, reação de câmara anterior $2+$, pontos tópicos, sem seidel, afacia (Figura 2).

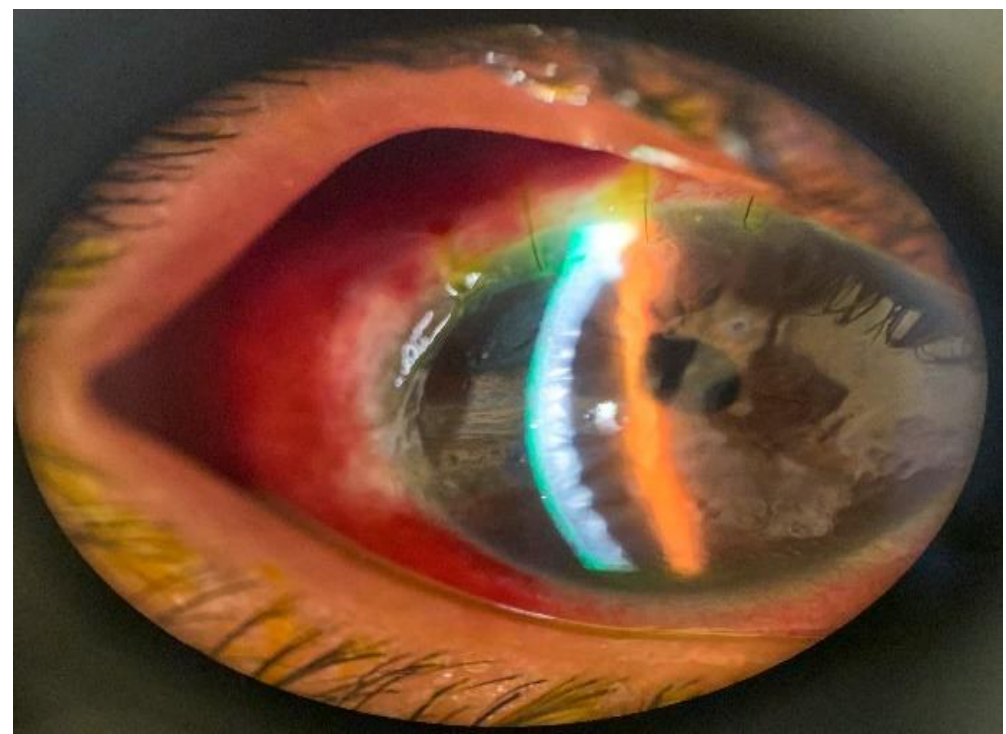

Figura 2. Primeiro dia de pós operatório após tratamento de urgência (facectomia sem implante de LIO). Fonte: $\mathrm{O}$ autor.

No sétimo dia de pós-operatório apresentou conjuntiva clara, córnea transparente, pontos tópicos, sem seidel, câmara anterior formada e profunda, sem reação de câmara anterior, afácica. A AV com correção estava $20 / 70$.

No final do primeiro mês de acompanhamento ambulatorial a paciente encontrava-se com reestabelecimento significativo da acuidade visual prévia a cirurgia no olho relatado, apresentando AV com correção de 20/50. Dessa forma, aguarda cirurgia de implante secundário de LIO em OD.

\section{DISCUSSÃO}

O presente relato de caso demonstrou que pacientes com SMF precisam de um acompanhamento interdisciplinar, com destaque para a avaliação oftalmológica periódica. Foi apresentado a resolução de um caso inesperado de luxação de cristalino para a câmara anterior, atraumático, após o mesmo estar luxado para a cavidade vítrea.

O estudo de Maumenee et al., em 1981, é um artigo de referência para características oculares em um estudo ocular com 160 pessoas portadoras de $\mathrm{SMF}^{15}$ de acordo com os critérios de 1979. ${ }^{4}$ Entre os 320 olhos, 193 mostraram subluxação do cristalino.
A subluxação do cristalino, também denominada "ectopia lentis", é a manifestação ocular mais comumente encontrada na SMF. ${ }^{16}$ Refere-se ao deslocamento do cristalino da sua posição normal no eixo visual, podendo estar luxado ou subluxado. Cerca de $60 \%$ dos casos apresentam subluxação do cristalino. $^{7}$ É usualmente bilateral e o cristalino pode estar deslocado em qualquer direção, sendo que em $77 \%$ dos casos encontra-se deslocado para cima, nasal superior ou temporal superior. ${ }^{17}$ Neste estudo, não foi observado subluxação do cristalino, mas sim a luxação para a câmara anterior. A luxação do cristalino para o segmento anterior ou posterior consiste em uma complicação relativamente incomum, mas potencialmente séria nestes pacientes. ${ }^{18}$

Quando o cristalino é deslocado totalmente, caracteriza-se a luxação. Essa locomoção para o espaço vítreo é rara e pode complicar-se em glaucoma facolítico. ${ }^{20} \mathrm{~A}$ luxação para a câmara anterior também é pouco frequente e pode produzir glaucoma agudo por bloqueio pupilar, ${ }^{19}$ como no presente caso.

Há poucos trabalhos que relatam a luxação do cristalino para o segmento anterior ou posterior na SMF. ${ }^{21}$ Drolsum L. et al. estudaram as características oculares em oitenta e sete pacientes com SMF e apenas um paciente 
desenvolveu glaucoma secundário em um dos olhos após a luxação do cristalino para câmara anterior, porém este não se localizava na cavidade vítrea, como no presente caso. ${ }^{21}$ Não foram encontrados trabalhos na literatura que descrevessem um paciente com SMF complicado com o cristalino luxado para a cavidade vítrea e que, antes do procedimento cirúrgico eletivo (VVPP), de forma espontânea, se deslocasse para a câmara anterior.

Estudos populacionais sugerem um comprimento axial normal em torno de $23,5 \mathrm{~mm}$ com base em medições do ultrassom A-Scan. ${ }^{22}$ Rand-Hendriksen et al. mostraram em seu estudo que o comprimento axial estava aumentado em $71 \%$ dos pacientes. ${ }^{23} \mathrm{~A}$ medida do comprimento axial dois meses antes do quadro relatado era de $26,39 \mathrm{~mm}$, também aumentada e considerada como um critério menor de Ghent- $1 .{ }^{4}$

O descolamento de retina é relatado como ocorrendo em cerca de $4 \%$ a $15 \%$ dos pacientes com SMF; ${ }^{16}$ e na maioria dos casos está relacionado ao comprimento axial e cirurgia envolvendo o cristalino. No presente estudo, não houve descolamento de retina antes ou após o procedimento.

No caso relatado, a indicação de cirurgia foi imperativa devido a hipertensão ocular causada pelo cristalino luxado para a câmara anterior, devendo ser retirado com urgência, assim como descrito na literatura. ${ }^{1}$ Nessa situação, antes da intervenção cirúrgica, o tratamento clínico deve ser tentado; tentativa sem sucesso no presente caso. Este consiste na realização de manobras de dilatação de posicionamento do paciente de rosto para cima, para que o cristalino volte para a câmara posterior, e, depois, utilizar miótico para fechar a pupila, para que o cristalino não volte a se deslocar anteriormente. Iridotomia a laser pode ser indicada nessa situação, mas deve-se priorizar a cirurgia para retirada do cristalino luxado, ${ }^{1}$ que foi a primeira opção nesse caso.

\section{CONCLUSÃO}

As alterações oculares da SMF são frequentes e o conhecimento da sua expressão no olho auxiliam o diagnóstico e tratamento destas anomalias. Este trabalho identificou uma complicação rara, de luxação do cristalino para o vítreo, na qual, antes do procedimento cirúrgico eletivo (VVPP), o próprio cristalino, de forma espontânea, deslocou-se para a câmara anterior.
A SMF trata-se de uma doença genética rara de elevada morbilidade. Os atuais recursos diagnósticos e terapêuticos permitiram não só a sua melhor compreensão patogênica, mas sobretudo otimizar de forma marcante o seu prognóstico.

O resultado do quadro clínico é substancialmente melhor quanto mais cedo for a extração do material cristaliniano luxado para a câmara anterior nos pacientes portadores da SMF, a fim de evitar as consequências (inclusive a cegueira) que a hipertensão ocular secundária pode ocasionar no nervo óptico e na retina.

\section{CONFLITO DE INTERESSE}

Os autores declaram não haver conflito de interesse que poderiam constituir um impedimento para a publicação deste artigo.

\section{REFERÊNCIAS}

1- Conselho Brasileiro de Oftalmologia CBO. Cristalino e Catarata. 4. ed. Rio de Janeiro: Cultura Médica; 2018. (Série Oftalmologia Brasileira).

2- Coelho SG, Almeida AG. Marfan syndrome revisited: From genetics to the clinic. Rev. port. cardiol. 2020;39(4):215-226. DOI: https://doi.org/10.1016/j.repc.2019.09.008

3- $\quad$ Sandvik GF, Vanem TT, Rand-Hendriksen S, Cholidis S, Saethre M \& Drolsum L. (2018). Tenyear reinvestigation of ocular manifestations in Marfan syndrome. Clin. Experiment. Ophthalmol. 2019; 47: 212- $218 . \quad$ DOI: https://doi.org/10.1111/ceo.13408

4- Loeys BL, Dietz HC, Braverman AC, Callewaert BL, De Backer J, Devereux RB et al. The revised Ghent nosology for the Marfan syndrome. J Med Genet. 2010;47(7):476-485. DOI: https://doi.org/10.1136/jmg.2009.072785

5- $\quad$ Suwal R, Khadka S \& Joshi P.. Ocular Manifestations and Biometrics in Marfan's Syndrome from Eastern Nepal. Clinical ophthalmology (Auckland, N.Z.). 2020 Aug. 25;14:2463-2472. DOI:

\section{https://doi.org/10.2147/OPTH.S269364}

6- $\quad$ Wilczyński TK, Niewiem A, Leszczyński R, Michalska-Małecka K. Recurrent Intraocular Lens Dislocation in a Patient with Familial Ectopia Lentis. Int. j. environ. res. public health. 2021 
Apr.25;18(9):4545.

DOI: https://doi.org/10.3390/ijerph18094545

7- Jones JA, Ikonomidis JS. The pathogenesis of aortopathy in Marfan syndrome and related diseases. Curr Cardiol Rep.2010;12:99-107. DOI: https://doi.org/10.1007/s11886-010-0083-z

8- Judge DP, Dietz HC. Marfan's syndrome. Lancet. 2005;366(9501):1965-1976. DOI: https://doi.org/10.1016/S0140-6736(05)67789-6

9- $\quad$ Beighton $P$, de Paepe A, Danks D, Finidori G, Gedde-Dahl T, Goodman R et al. International Nosology of Heritable Disorders of Connective Tissue, Berlin, 1986. Am J Med Genet. 1988;29: 581-594.

DOI:

https://doi.org/10.1002/ajmg.1320290316

10- Fuchs J, Rosenberg T. Congenital ectopia lentis. A Danish national survey. Acta Ophthalmol Scand. 1988;76: 20-26. DOI: https://doi.org/10.1034/j.1600-

$\underline{0420.1998 .760105 . x}$

11- Esfandiari $\mathrm{H}$, Ansari S, Mohammad-Rabei $\mathrm{H}$, Mets MB. Management Strategies of Ocular Abnormalities in Patients with Marfan Syndrome: Current Perspective. Journ. ophth. vision research. 2019;14(1):71-77. DOI: https://doi.org/10.4103/jovr.jovr $29 \quad 18$

12- American Academy of Ophthalmology. Basic and Clinical Science Course, Section 6: Pediatric Ophthalmology and Strabismus. San Francisco: American Academy of Ophthalmology; 2014.

13- Anteby I, Isaac M, BenEzra D. Hereditary subluxated lenses visual performances and longterm follow-up after surgery. Ophthalmology. 2003;110: 1344-8. DOI: https://doi.org/10.1016/S0161-6420(03)00449-4

14- Denise EH, Eduardo SS, Rodrigo LM, Gustave NA, Walton N. Outcomes of Iris-Claw Anterior Chamber versus Iris-Fixated Foldable Intraocular Lens in Subluxated Lens Secondary to Marfan Syndrome. Ophthalmology. 2010;117: 1479-85. DOI: https://doi.org/10.1016/i.ophtha.2009.12.043

15- Maumenee $\mathrm{IH}$. The eye in Marfan syndrome. Trans Am Ophthalmol Soc.
1981;79:684-733.

16- Chandra A, Ekwalla V, Child A, Charteris D. Prevalence of ectopia lentis and retinal detachment in Marfan syndrome. Acta Ophthalmol. 2014;92: e82-e83. DOI: https://doi.org/10.1111/aos.12175

17- Perez ABA. Anomalias oculares e características genéticas na Síndrome de Marfan. Arq Bras Oftalmol. 2002;65:623-8. DOI: https://doi.org/10.1590/S000427492002000600005

18- Konradsen TR, Zetterstrom C. A descriptive study of ocular characteristics in Marfan syndrome. Acta Ophthalmol. 2013;91:751-755. DOI: https://doi.org/10.1111/aos.12068

19- Lambrou FH Jr, Stewart MW. Management of dislocated lens fragments during phacoemulsification. Ophthalmology. 1992;99(8):1260-2. DOI: https://doi.org/10.1016/S0161-6420(92)31805-6

20- Mandia Jr C, Almeida GV, Almeida PB, Cohen R. Glaucoma facolítico. In: Almeida HG, Cohen R. Glaucomas secundários. 2. ed. São Paulo: Roca; 2005.

21- Drolsum L, Rand-Hendriksen S, Paus B, Geiran OR, Semb SO. Ocular findings in 87 adults with Ghent-1 verified Marfan syndrome. Acta Ophthalmol 2015;93(1):46-53. DOI: https://doi.org/10.1111/aos.12448

22- Olsen T, Arnarsson A, Sasaki H, Sasaki K \& Jonasson F. On the ocular refractive components: the Reykjavik Eye Study. Acta Ophthalmol Scand. 2007;85:361-366.

DOI:

https://doi.org/10.1111/j.1600-

$\underline{0420.2006 .00847 . x}$

23- Rand-Hendriksen S, Lundby R, Tjeldhorn L., Andersen K., Offstad J, Semb SO et al. Prevalence data on all Ghent features in a crosssectional study of 87 adults with proven Marfan syndrome. Eur J Hum Genet. 2009;17:1222-1230. DOI: https://doi.org/10.1038/ejhg.2009.30 